\title{
A UTILIZAÇÃO DO ENADE COMO INSTRUMENTO DE AVALIAÇÃo EM CURSOS DE ENGENHARIA CIVIL
}

Leonardo C. Machado - leonardocmachado96@usp.br

Daniel A. Souza - d.souza@ usp.br

Welles J. Oliveira - welles.oliveira@usp.br

Departamento de Engenharia de Construção Civil, Escola Politécnica da USP

R. Prof. Almeida Prado, travessa 1, 115

05508-070 - São Paulo - SP

Resumo: No Brasil o Exame Nacional de Desempenho dos Estudantes (ENADE) é uma ferramenta utilizada para avaliar os cursos em instituições de ensino superior. Este trabalho faz uma análise crítica do ENADE para o curso de Engenharia Civil no ano de 2017 comparando a prova e os relatórios de curso de três instituições públicas de ensino superior: Instituto Federal de São Paulo, Universidade Federal de Alagoas e Universidade Federal de Uberlândia. A análise foi feita considerando a composição da prova, o desempenho dos alunos das instituições na prova e perfil socioeconômico dos alunos.

Palavras-chave: ENADE. Engenharia Civil. Ensino Superior.

\section{INTRODUÇÃO}

A instituição de ensino superior é responsável pela formação do engenheiro civil e tem por objetivo dotar ao profissional conhecimentos requeridos em competências e habilidades específicas. conforme especificado pela Resolução CNE/CES n 2, de 24 de abril de 2019 Diretrizes Curriculares Nacionais do Curso de Graduação em Engenharia, apresentados na Tabela 1.

Tabela 1 - Competências e habilidades propostas pelo ENADE/SINAES para o curso de Engenharia Civil

\begin{tabular}{|l|l|}
\hline I & $\begin{array}{l}\text { Formular e conceber soluções desejáveis de engenharia, analisando e compreendendo os usuários dessas } \\
\text { soluções e seu contexto }\end{array}$ \\
\hline II & $\begin{array}{l}\text { Analisar e compreender os fenômenos físicos e químicos por meio de modelos simbólicos, físicos e } \\
\text { outros, verificados e validados por experimentação }\end{array}$ \\
\hline III & Conceber, projetar e analisar sistemas, produtos (bens e serviços), componentes ou processos \\
\hline IV & Implantar, supervisionar e controlar as soluções de engenharia \\
\hline V & Comunicar-se eficazmente nas formas escrita, oral e gráfica \\
\hline VI & Trabalhar e liderar equipes multidisciplinares \\
\hline VII & Conhecer e aplicar com ética a legislação e os atos normativos no âmbito do exercício da profissão \\
\hline VIII & $\begin{array}{l}\text { Aprender de forma autônoma e lidar com situações e contextos complexos, atualizando-se em relação } \\
\text { aos avanços da ciência, da tecnologia e aos desafios da inovação }\end{array}$ \\
\hline
\end{tabular}


No Brasil, o Exame Nacional de Desempenho dos Estudantes (ENADE) é uma ferramenta de avaliação do Sistema Nacional de Avaliação da Educação Superior (SINAES). Além do ENADE, os processos Avaliação de Cursos de Graduação e Avaliação Institucional constituem o tripé avaliativo do SINAES. Os resultados desses instrumentos avaliativos, reunidos, permitem conhecer, em profundidade, o modo de funcionamento e a qualidade dos cursos e instituições de educação superior (IES) de todo o País.

$\mathrm{O}$ curso de Engenharia Civil abrange uma diversidade de conhecimento. No curso o estudante deve ser capacitado a aplicar novas tecnologias, gerenciar, criar projetos, construir e inspecionar. Para isto, os exames aplicados no processo de ensino de engenharia devem abordar a organização racional dos conteúdos (GAO, 2011).

As estratégias de ensino de cada instituição de ensino podem variar conforme a região do país. Por conseguinte, este trabalho tem como objetivo principal analisar o ENADE no curso de Engenharia Civil em três instituições públicas diferentes no ano de 2017. As instituições de ensino escolhidas foram instituições da formação na graduação dos autores: Instituto Federal de São Paulo - IFSP, Universidade Federal de Alagoas - UFAL e Universidade Federal de Uberlândia - UFU.

\section{METODOLOGIA}

A análise do ENADE para o curso de Engenharia Civil no ano de 2017 foi realizada comparando a prova e os relatórios de curso de três instituições públicas de Ensino Superior: IFSP, UFAL E UFU. A análise foi feita considerando a composição da prova, o desempenho dos alunos das instituições na prova e perfil socioeconômico dos alunos.

\subsection{Composição e avaliação da prova}

A prova do Enade para o curso de Engenharia Civil no ano de 2017 foi avaliada pela quantidade de questões (originais e válidas), o tempo médio de resolução e a dificuldade média das questões por área de conhecimento. O tempo e dificuldade das questões foi escalado de acordo com a Tabela 3 e as áreas de conhecimento foram divididas conforme a Tabela 4.

Tabela 3 - Escalas de dificuldade e tempo de resolução das questões

\begin{tabular}{|c|c|c|c|}
\hline Nível de dificuldade & Escala & Tempo para resolução das questões & Escala \\
\hline Fácil & 1 & Curto & 1 \\
\hline Média & 2 & Médio & 2 \\
\hline Difícil & 3 & Longo & 3 \\
\hline
\end{tabular}


Tabela 4 - Divisão das áreas de conhecimento - formação específica e geral

\begin{tabular}{|c|c|}
\hline Área de conhecimento - Formação específica & Identificação \\
\hline Materiais de Construção e Ciência dos Materiais & 1 \\
\hline Hidráulica e Fenômeno dos Transportes & 3 \\
\hline Resistência dos Materiais, Estruturas e Fundações & 4 \\
\hline Transportes e Solos & 5 \\
\hline Construção Civil & 6 \\
\hline Sustentabilidade e Resíduos & 8 \\
\hline Administração e Economia & 9 \\
\hline Elétrica & Identificação \\
\hline Outros & 10 \\
\hline Área de conhecimento - Formação geral & 11 \\
\hline Anterpretação de Texto / Redação / Problemas Sociais & 12 \\
\hline Geografia & \\
\hline
\end{tabular}

\subsection{Desempenho das instituições por área de conhecimento}

O desempenho das instituições de ensino público superior IFSP, UFAL e UFU foi analisado e comparado conforme a divisão de áreas de conhecimento. Para isso, foi avaliado o percentual de acerto dos alunos e calculadas as médias gerais.

Para os resultados da prova do ENADE de 2017 considerou-se como acerto as questões anuladas na prova. E para cálculo da média geral adotou-se fator de 0,75 para as questões de conhecimento específico e 0,25 para as questões de conhecimento geral.

\subsection{Caracterização socioeconômica}

A caracterização socioeconômica dos alunos do curso de Engenharia Civil das instituições analisadas no ENADE de 2017 foi realizada observando a representatividade étnica, a renda familiar, a renda individual e o perfil de ingresso no ensino superior adotando o critério de cotas. 


\section{RESULTADOS E DISCUSSÕES}

\subsection{Composição e avaliação da prova}

Em análise da composição da prova do ENADE de 2017 para o curso de Engenharia Civil, verificou-se que das 40 questões originais, apenas 32 questões foram consideradas como válidas. Desta forma, é possível perceber falhas na elaboração da prova, uma vez que, $20 \%$ das questões foram anuladas. A Figura 1 mostra os dois cenários por área de conhecimento: questões originais e questões válidas.

Figura 1 - Quantidade de questões por área de conhecimento

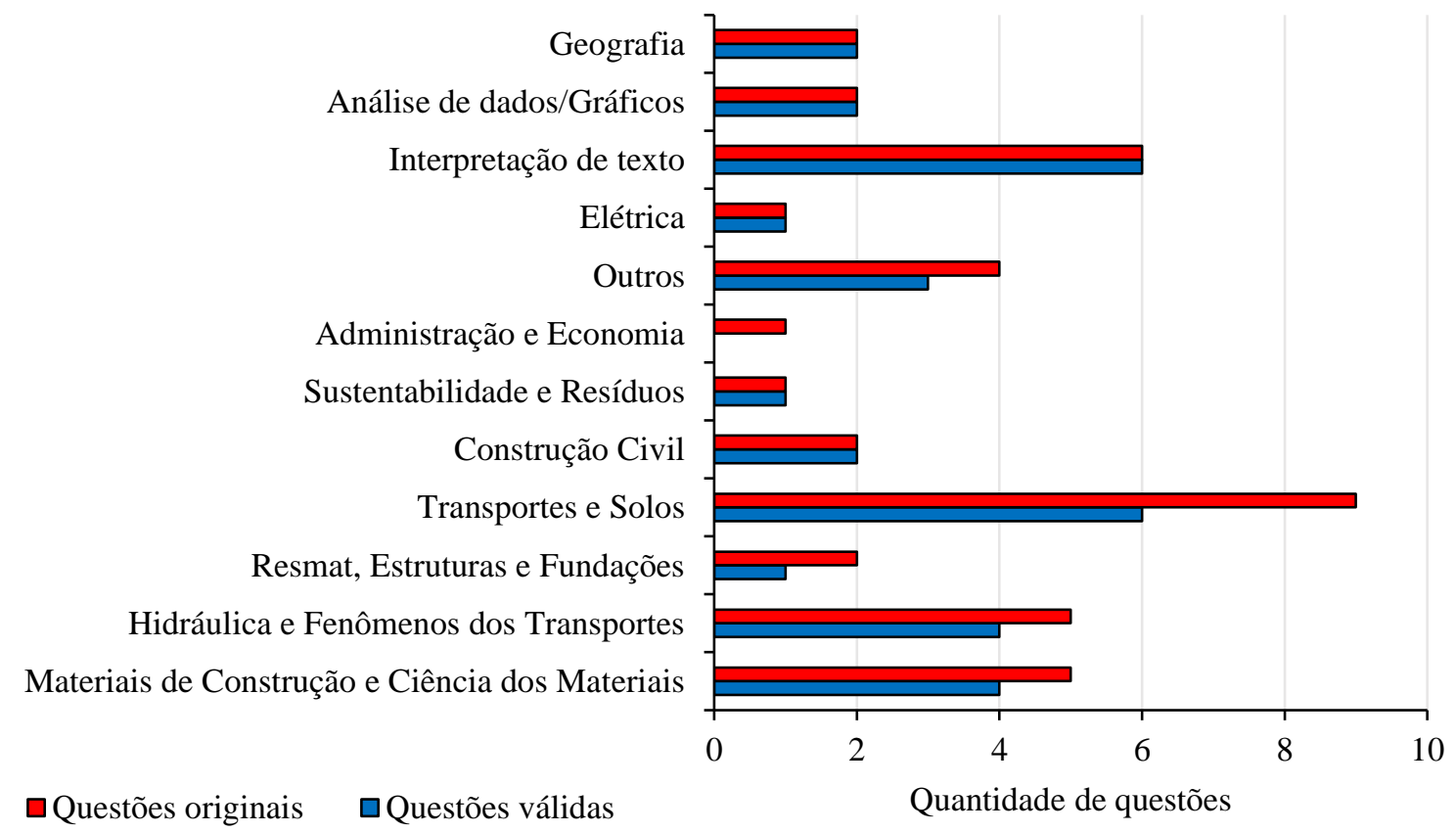

Na divisão da prova por área de conhecimento há discrepância na quantidade de questões. A área de Transportes e Solos, por exemplo, apresentou a maior quantidade de questões, no total de nove questões no cenário original. Enquanto a área de Resistência dos Materiais (Resmat), Estruturas e Fundações tiveram apenas duas questões, da qual apenas uma foi válida. Ressalta-se que estas duas áreas são de conhecimento específico, e grande importância para o curso de Engenharia Civil.

Em avaliação da prova do ENADE deve-se atentar a outros dois fatores: tempo médio de resolução de questão e dificuldade média das questões. Estes fatores são descritos na Figura 2 para cada área de conhecimento na prova para o curso, levando em conta a escala de 1 a 3 , descrita no item 2.1 da metodologia.

$\mathrm{Na}$ maior parte das áreas de conhecimento da prova o tempo médio de resolução das questões está na escala de tempo entre curto e médio; em exceção da área de Sustentabilidade e Resíduos e a área de Resistência dos Materiais, Estruturas e Fundações que apresentam tempo de resolução médio e longo, respectivamente.

No quesito dificuldade média das questões, em geral, as áreas de conhecimento foram categorizadas entre fácil e média; em exceção também a área de Sustentabilidade e Resíduos e a área de Resistência dos Materiais, Estruturas e Fundações que possuíram dificuldade média e difícil, respectivamente. 


\section{Sa COPENCE C. COBENGE 2020 da ABENGE}

"Os desafios para formar hoje o engenheiro do amanhã"

Figura 2 - Tempo médio e dificuldade média das questões da prova por área de conhecimento

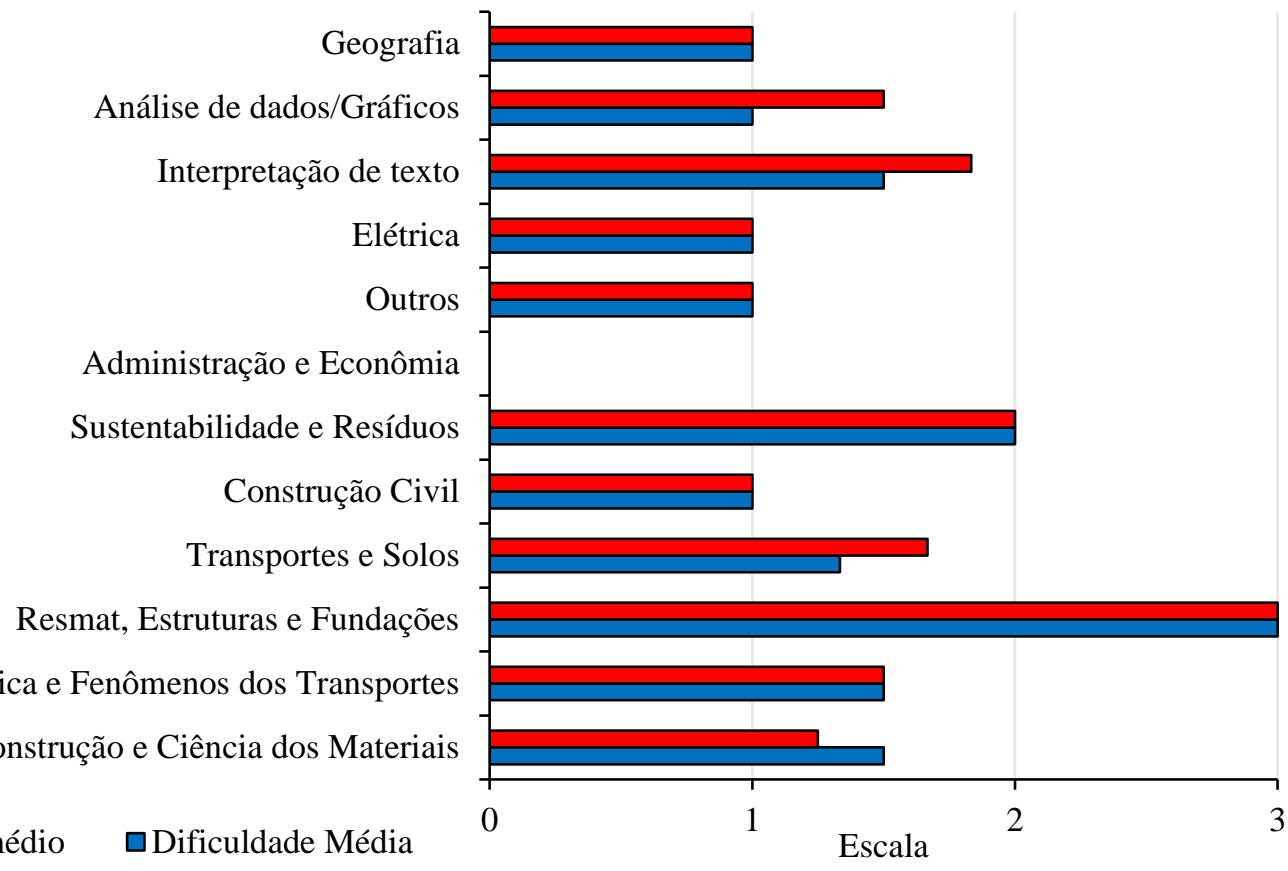

\subsection{Desempenho das instituições por área de conhecimento}

A Figura 3 apresenta o percentual médio de acertos dos estudantes por área de conhecimento para as instituições de ensino superior público IFSP, UFAL e UFU na prova do ENADE de 2017 para o curso de Engenharia Civil. Em geral, as instituições de ensino apresentaram percentuais próximos para a maior parte das áreas de conhecimento, em exceção das áreas de Geografia e Elétrica em que a UFAL se mais destacou dentre as outras instituições.

Figura 3 - Percentual de acertos por área de conhecimento para as instituições IFSP, UFAL e UFU

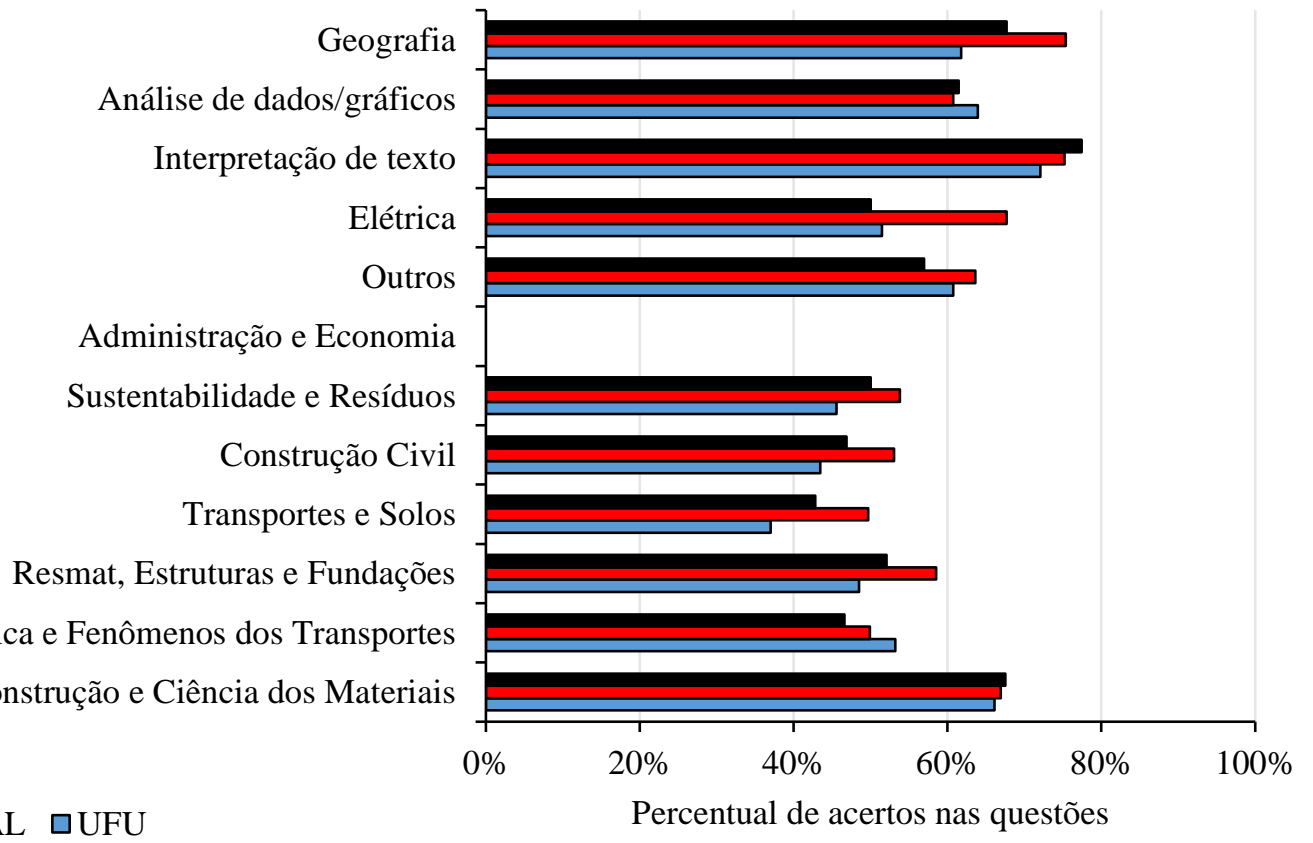


C COBENCE 2020

"Os desafios para formar hoje o engenheiro do amanhã"
$\mathrm{Ol}$ a $\mathrm{O3}$ de dezembro Evento On-line

A área Materiais de Construção e Ciência dos Materiais apresentou percentual de acertos mais próximo entre as instituições de ensino analisadas, e é a área de conhecimento específico com melhor resultado, tendo sido abordada em quatro das questões válidas da prova do ENADE de 2017.

O percentual de questões acertadas pelos estudantes das instituições em conhecimentos gerais e conhecimentos específicos e a média geral na prova do ENADE de 2017 para o curso de Engenharia Civil, são apresentados na Figura 4. As questões anuladas foram consideradas como corretas pelo ENADE. A média geral foi calculada ponderando peso de 0,75 e 0,25 para questões de conhecimento específico e conhecimento gerais, respetivamente. Desta forma, a UFAL foi a instituição pública que mais se destacou na média, sobressaindo-se nas questões de conhecimento específico, as quais possuem maior peso no cálculo da média.

Figura 4 - Resultados da prova do ENADE de 2017 nas instituições IFSP, UFAL e UFU

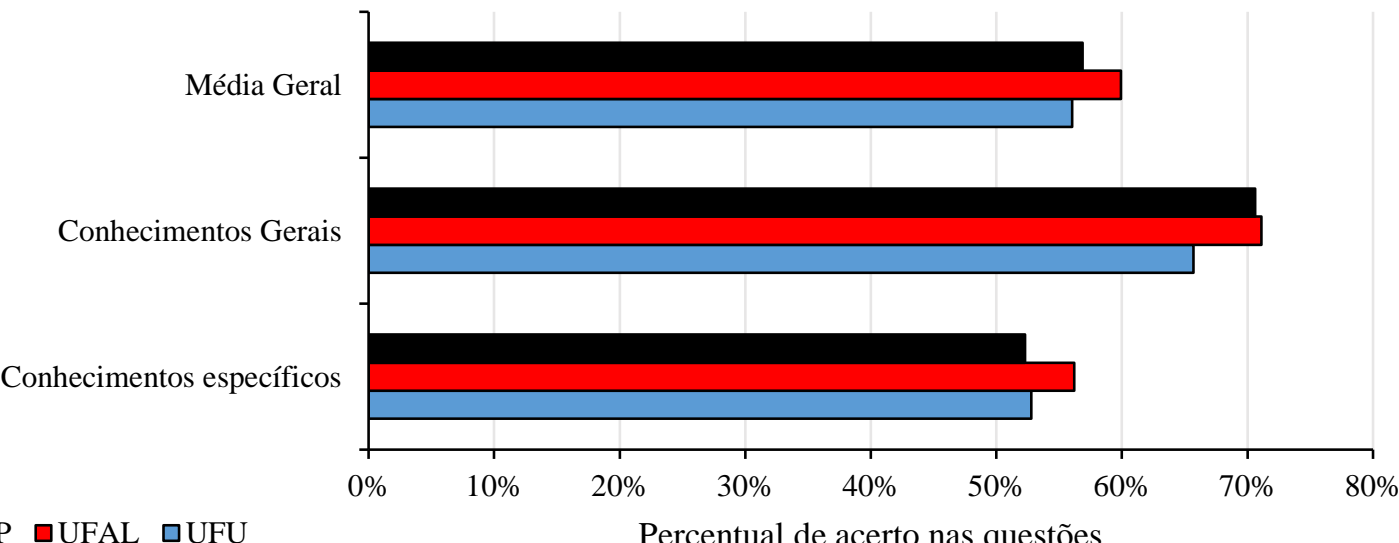

-IFSP 口UFAL 口UFU

Percentual de acerto nas questões

\subsection{Caracterização socioeconômica}

De maneira geral, não se observa representatividade indígena nas instituições de ensino superior avaliadas, o que já era esperado visto que todas as instituições são localizadas em áreas de menor população indígena. Ressalta-se a predominância da etnia branca em relação às demais (FIGURA 5). A UFAL foi a instituição com maior equilíbrio e diversidade étnica.

Figura 5 - Representatividade étnica dos estudantes de engenharia civil nas instituições

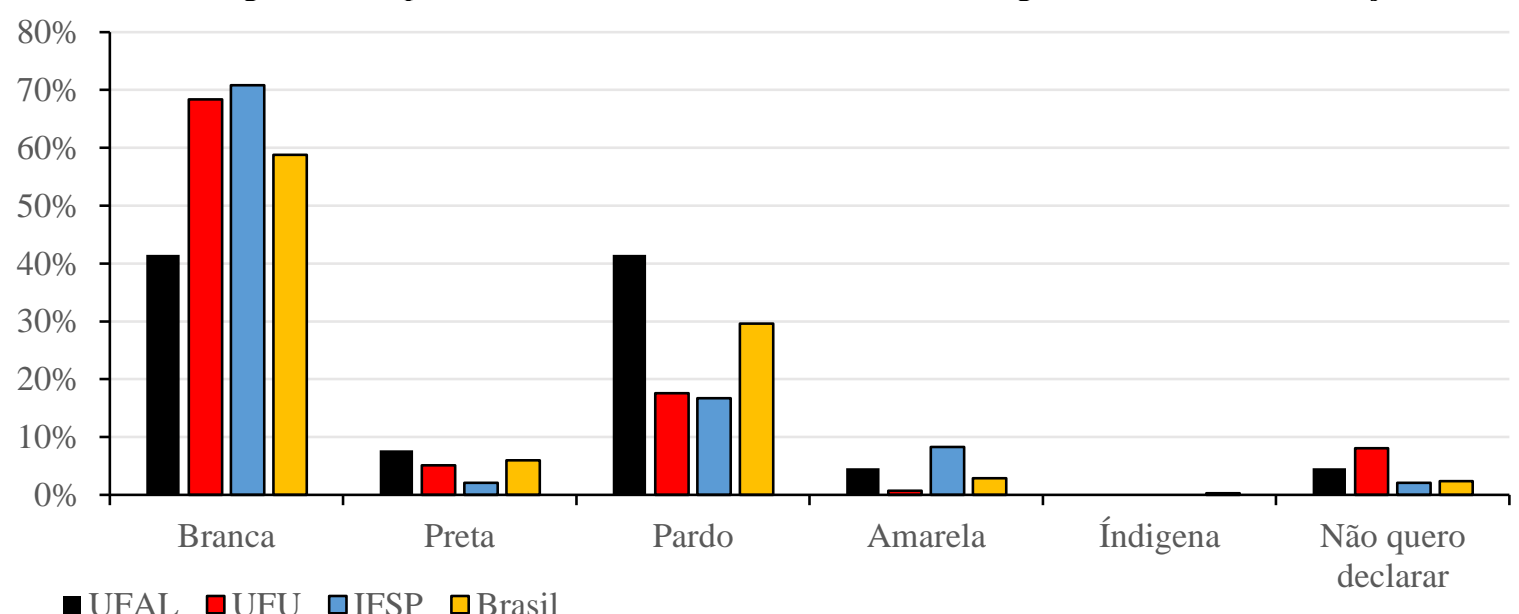


Figura 7 - Renda individual dos estudantes de engenharia civil nas instituições

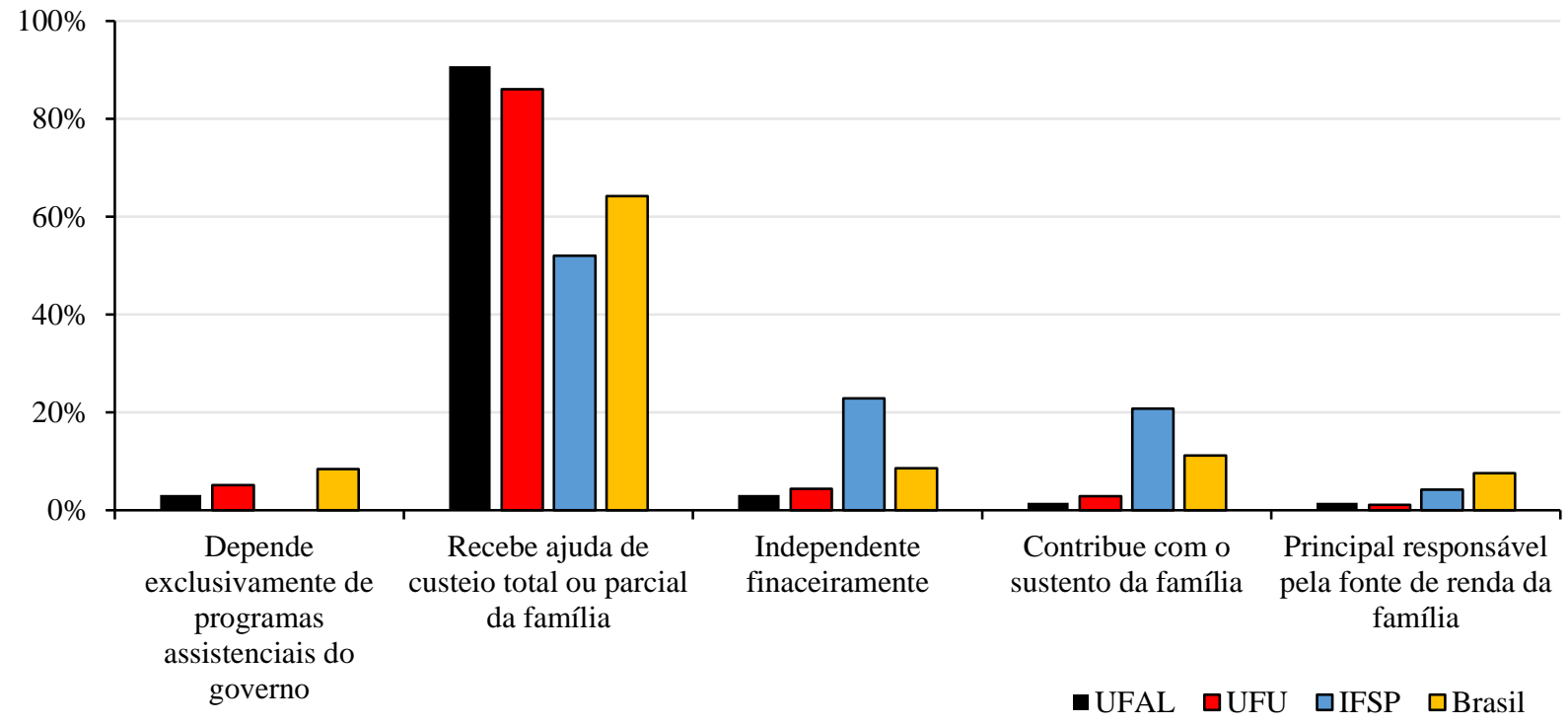

Observa-se ainda uma baixa aderência das instituições brasileiras ao ingresso pelo sistema de cotas, como visto na Figura 8. Dentre às universidades avaliadas a UFU apresentou o maior índice de alunos cotistas, com cerca de $34 \%$ dos alunos provenientes de alguma ação afirmativa de cotas. Esse quadro é ainda mais grave ao se considerar os números da UFAL, visto que apenas 16,9\% dos alunos ingressantes são cotistas, ou seja, para cada aluno cotista que adentra o sistema, cinco alunos são não cotistas, o que contribui para a desigualdade no estado de Alagoas, visto que a UFAL é a principal instituição pública do estado.

Figura 8 - Perfil de ingresso dos estudantes de engenharia civil nas instituições - critério de cotas

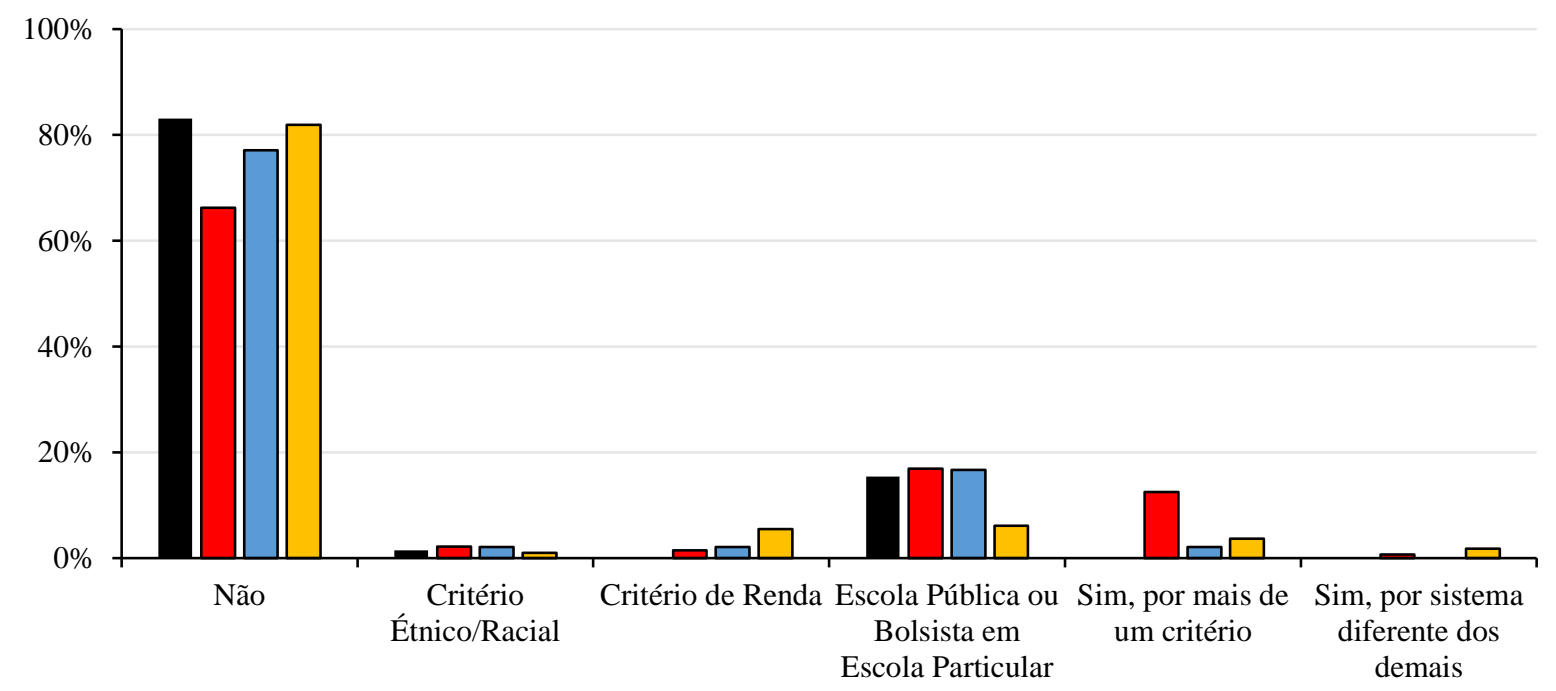

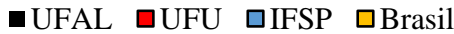




\section{CONCLUSÕES}

A prova do ENADE para o curso de Engenharia Civil no ano de 2017 apresentou irregularidades como ferramenta de avaliação do curso. A prova não demonstrou uniformidade na distribuição das questões por área de conhecimento, focando mais na área de Transportes e Solos, área na qual teve a maior quantidade de questões anuladas. Os autores questionam a eficácia de avaliar o curso com um número pequeno de questões, uma vez que, em áreas como Estruturas, errar uma única questão representaria 50\% da área. Além disso, 20\% das questões totais da prova foram anuladas, o que indica a necessidade de um melhor cuidado no planejamento e elaboração. Acredita-se que, um modelo com mais questões e distribuição uniforme das questões nas áreas de conhecimento, como o que é feito em provas da FUVEST e do ENEM, seria mais eficaz para a avaliação.

O relatório do ENADE não apresenta a análise por área de conhecimento feita no presente artigo. Esta análise crítica poderia fornecer subsídios para os coordenadores e diretores de curso intervirem em áreas de conhecimento com maior deficiência, visando a melhoria contínua e o objetivo maior, que é o aprendizado do aluno.

Dentre as três instituições públicas de ensino superior, a UFAL apresentou melhor média geral no ENADE de 2017 para o curso de Engenharia Civil, destacando-se nas áreas de conhecimento específico. A UFAL é a instituição com mais alunos que possuem renda familiar acima de 10 salários mínimos e com maior percentual de alunos que recebem ajuda de custeio da família.

Por conseguinte, a UFAL foi a instituição que apresentou maior variedade de etnias, entretanto, apresentou a maior desigualdade econômica entre as instituições analisadas e o menor número de alunos cotistas. A UFU foi a instituição que melhor aderiu o sistema de cotas no curso de Engenharia Civil. E, a IFSP apresentou a maior quantidade de alunos independentes financeiramente.

\section{REFERÊNCIAS}

CONSELHO FEDERAL DE ENGENHARIA E AGRONOMIA. Resolução $\mathrm{N}^{\circ} 1.073$ Atividades e competências profissionais do engenheiro de energia. CONFEA/CREA, 2016.

CONSELHO NACIONAL DE EDUCAÇÃO SUPERIOR. Resolução CNE/CES n² Diretrizes Curriculares Nacionais do Curso de Graduação em Engenharia. MEC, 2019.

GAO, B. Study on the engineering practice course education teaching reform. Procedia Engineering, v. 15, p. 4224-4227, 2011.

INSTITUTO NACIONAL DE ESTUDOS E PESQUISAS EDUCACIONAIS ANÍSIO TEIXEIRA. Relatório de Curso: Engenharia Civil - Enade 2017. Universidade Federal de Alagoas. INEP, 2018.

INSTITUTO NACIONAL DE ESTUDOS E PESQUISAS EDUCACIONAIS ANÍSIO TEIXEIRA. Relatório de Curso: Engenharia Civil - Enade 2017. Universidade Federal de Uberlândia. INEP, 2018.

INSTITUTO NACIONAL DE ESTUDOS E PESQUISAS EDUCACIONAIS ANÍSIO TEIXEIRA. Relatório de Curso: Engenharia Civil - Enade 2017. Instituto Federal de São Paulo. INEP, 2018. 


\title{
ANALYSIS OF ENADE AS A TOOL TO EVALUATE CIVIL ENGINEERING COURSES
}

\begin{abstract}
The National Assessment of Student Achievement - ENADE (in Portuguese) is one of the main tools Brazil used to assess the quality of undergraduate courses throughout the country. The programs are grouped in three representative areas and each year one group is assessed, meaning that each program is assessed every three years. This paper focuses on analyzing the efficacy of ENADE in assessing civil engineering courses, for that three very distinctive universities are selected, one in the countryside (UFU), one in the northeast region (UFAL) and one located in São Paulo city (IFSP). The Analysis considers as main parameters, the composition of the exam, the performance of each institution and an overall socioeconomic profile of the students.
\end{abstract}

Keywords: Higher Education Quality. Undergraduate Courses, Civil Engineering. 